\title{
How to Find the Electron Starting Block
}

\section{A new technique pinpoints, with picometer resolution, the location from which an emitted electron originates within a molecule.}

\author{
By Michael Schirber
}

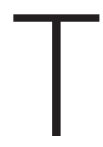
he inside of an atom or a molecule is a mix of overlapping electron orbitals. New experiments allowed researchers to peer into that complex environment and learn the starting location for an electron that was emitted by a nitrogen molecule, $\mathrm{N}_{2}$, during ionization [1]. The researchers used a common laser-based technique in which the electron quantum mechanically interferes with itself on its way out of the molecule. By detecting a small shift in the interference pattern, the team showed that the electron started 95 picometers away from the center of the molecule. The results could help researchers understand the migration of charge inside molecules during chemical reactions.

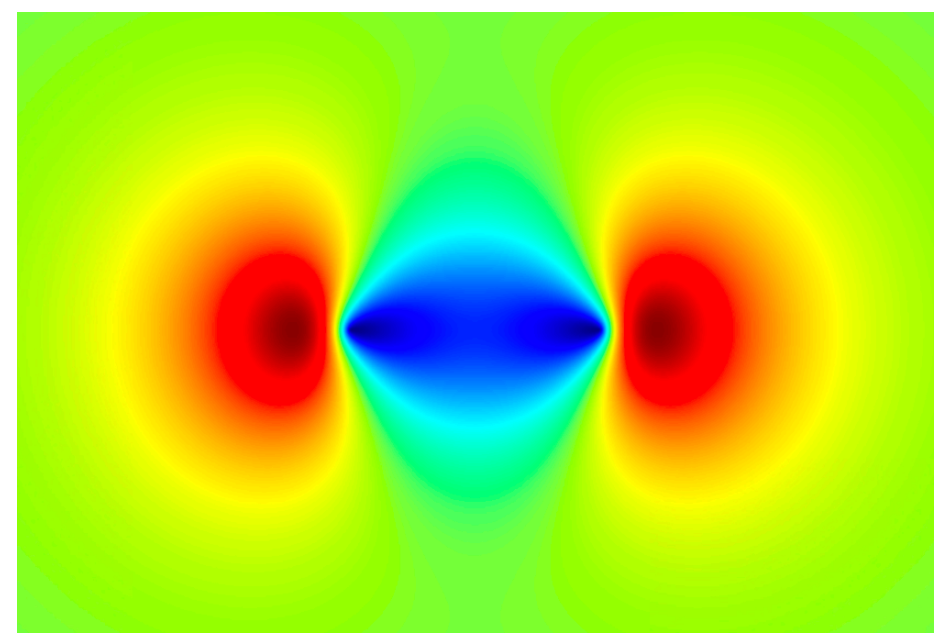

Coming out of the clouds. A new experiment tracks the emission of electrons from ionized nitrogen molecules, determining the electron starting location within the molecular orbitals (shown here).

Credit: W. Xie et al. [1]
Ionization occurs when an electron escapes past the potential energy barrier that surrounds an atom or molecule. In normal photoionization, the electron absorbs the energy of a photon (or multiple photons), acquiring enough kinetic energy to "climb" over the barrier. In tunneling ionization, a laser distorts the potential energy curve in a gradual process that lowers the barrier on one side of the atom or molecule. One of the electrons is then able to "tunnel" out, which means it crosses the barrier quantum mechanically.

Researchers are interested in tunneling ionization because it offers a way to reconstruct the motion of an ejected electron. Previous studies have, for example, revealed the position where the electron exits the tunnel, the time when it exits, and the typical speed with which it exits. Min Li from Huazhong University of Science and Technology in China and colleagues have now determined the electron's location before it enters the tunnel. Knowing the electron's origin is "more fundamental than the information obtained by previous studies," Li says.

Their experiment is based on the typical tunneling ionization setup, with a strong laser for ionizing the nitrogen gas and a detector for collecting the emitted electrons. In each nitrogen molecule, the departing electron takes one of two paths toward the detector: one path is direct, whereas the second path involves a short detour in which the electron scatters through the molecular center before heading to the detector. Those two paths result in an interference pattern in a plot of the measured electron momentum. Li and colleagues modified the usual experiment by adding a weak laser whose polarization (electric field direction) is perpendicular to that of the strong laser. This second laser causes a tiny shift in the fringes of the interference pattern. 


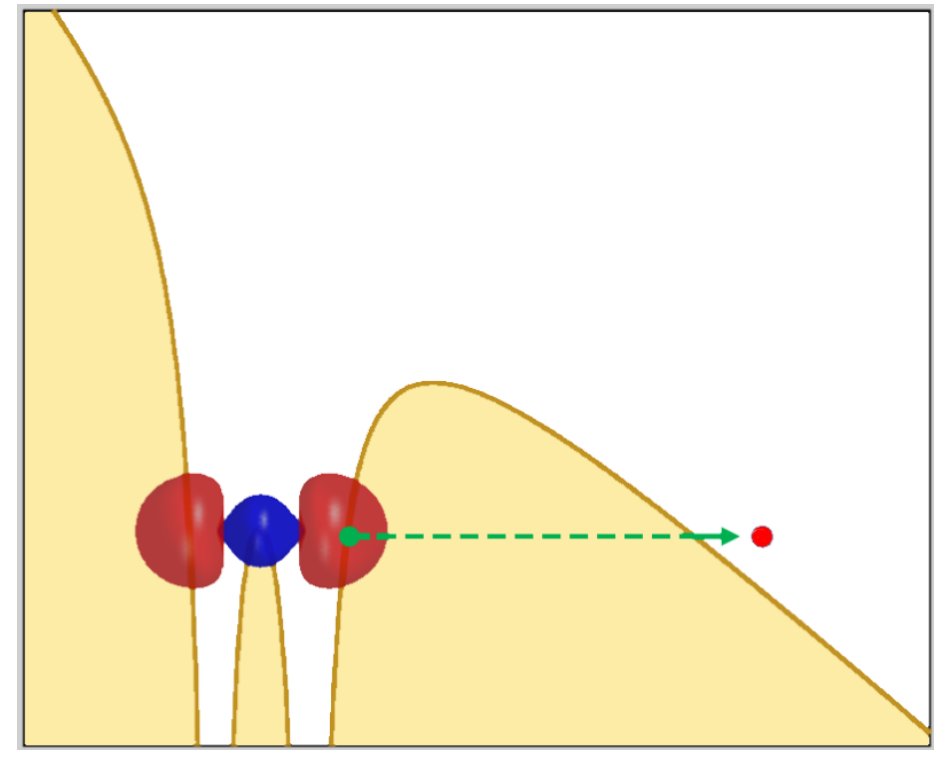

End of the tunnel. A laser causes the potential energy barrier (yellow) around a molecule to lower on one side. An electron in one of the molecule's outer orbitals (red clouds) is then able to tunnel through and escape from the molecule as a free particle. Credit: Adapted from W. Xie et al. [1]

Previously, the researchers had used this two-laser method with atoms to measure the momentum of the ionized electrons [2]. But now they have used it with molecules prior to ionization. The researchers used a short pulse of light to align the molecules so that all of their nitrogen-nitrogen bonds pointed in the same direction relative to the strong laser's polarization. They observed that the shift in the fringes depended on this alignment direction. By modeling the effect that the second laser has on the lengths of the direct and scattered paths, the team was able to convert the fringe data into a measurement of the starting point of the electron.

They found that the tunneling electrons were not located at the center of the molecule-as many simplifying models assume-but instead were $95 \pm 21$ picometers away from the center, along the axis defined by the nitrogen-nitrogen bond. For comparison, the two nitrogen nuclei are situated about 54 picometers from the center. The team showed that the electron's position agrees with computations of the highest occupied molecular orbital, which is the orbital requiring the least energy for ionization.

Tunneling ionization is often used in so-called attosecond science to perform, for example, high-harmonic spectroscopy and attoclock timing (see Viewpoint: Timing Molecular Motion with an Optical Stopwatch). "The measured photoemission position can be used to improve the precision of these attosecond metrologies," Li says. The team also plans to extend their method to more complex molecules. The mapping of photoemission from such molecules could benefit studies of charge migration-the movement of electrons during chemical reactions and biological processes.

"This work is certainly top-notch and provides unprecedented information about tunneling ionization," says Markus Zeiler from the Vienna University of Technology. "Experimental knowledge of the photoemission position is important because it allows gauging tunneling theories and therefore helps in improving them."

Michael Schirber is a Corresponding Editor for Physics based in Lyon, France.

\section{REFERENCES}

1. W. Xie et al., "Picometer-resolved photoemission position within the molecule by strong-field photoelectron holography," Phys. Rev. Lett. 127, 263202 (2021).

2. M. Li et al., "Photoelectron holographic interferometry to probe the longitudinal momentum offset at the tunnel exit," Phys.

Rev. Lett. 122, 183202 (2019). 